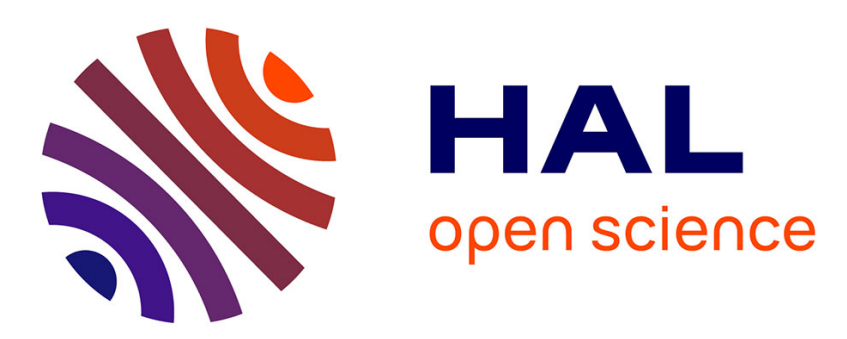

\title{
Plasticité du sesquioxyde d'yttrium (Y2O3) par micro-indentation
}

\author{
R.J. Gaboriaud, M. Boisson
}

\section{To cite this version:}

R.J. Gaboriaud, M. Boisson. Plasticité du sesquioxyde d'yttrium (Y2O3) par micro-indentation. Revue de Physique Appliquée, 1978, 13 (5), pp.219-221. 10.1051/rphysap:01978001305021900 . jpa00244443

\section{HAL Id: jpa-00244443 https://hal.science/jpa-00244443}

Submitted on 1 Jan 1978

HAL is a multi-disciplinary open access archive for the deposit and dissemination of scientific research documents, whether they are published or not. The documents may come from teaching and research institutions in France or abroad, or from public or private research centers.
L'archive ouverte pluridisciplinaire HAL, est destinée au dépôt et à la diffusion de documents scientifiques de niveau recherche, publiés ou non, émanant des établissements d'enseignement et de recherche français ou étrangers, des laboratoires publics ou privés. 
Classification

Physics Abstracts

$62.20 \mathrm{~F}$

\title{
PLASTICITÉ DU SESQUIOXYDE D'YTTRIUM $\left(\mathbf{Y}_{2} \mathbf{O}_{3}\right)$ PAR MICRO-INDENTATION
}

\author{
R. J. GABORIAUD et M. BOISSON \\ Laboratoire de Métallurgie Physique \\ 40, avenue du Recteur-Pineau, 86022 Poitiers, France
}

(Reçu le 8 novembre 1977, révisé le 16 janvier 1978, accepté le 18 janvier 1978)

\begin{abstract}
Résumé. - Une étude de la microdureté du sesquioxyde d'yttrium, $\mathrm{Y}_{2} \mathrm{O}_{3}$, en fonction de la température est réalisée par indentation Vickers. Une déformation plastique évidente apparaît à partir de $480^{\circ} \mathrm{C}\left(0,28 T_{\mathrm{f}}\right)$. Des empreintes faites sur une face (111) montrent une possibilité de plasticité dès la température ambiante.
\end{abstract}

Abstract. - Microhardness investigation of the yttrium sesquioxide $\left(\mathrm{Y}_{2} \mathrm{O}_{3}\right)$ against temperature is performed by means of a Vickers indenter. A clear plastic deformation appears at $480{ }^{\circ} \mathrm{C}$ $\left(0.28 T_{\mathrm{f}}\right)$. Tests carried out on a (111) face show a possible plasticity at room temperature.

1. Introduction. - Le sesquioxyde d'yttrium $\left(\mathrm{Y}_{2} \mathrm{O}_{3}\right)$ possède un certain nombre de propriétés technologiques intéressantes. Son haut point de fusion, $2723 \mathrm{~K} \mathrm{[1]} \mathrm{en} \mathrm{fait} \mathrm{un} \mathrm{excellent} \mathrm{réfractaire.} \mathrm{Sa} \mathrm{chaleur}$ de formation, une des plus élevée parmi celles des oxydes [2], lui confère une grande stabilité par rapport à la température. C'est un des oxydes les moins volatils [3].

$\mathrm{Y}_{2} \mathrm{O}_{3}$ est utilisé comme céramique mélangé à $10 \%$ de thorine $\left(\mathrm{ThO}_{2}\right)$ sous le nom d'yttralox $[4,5,6]$. Pressé à chaud, il devient transparent avec une densité très proche de la densité théorique $[6,7]$. Une application importante de $\mathrm{Y}_{2} \mathrm{O}_{3}$ provient du fait qu'il stabilise la zircone $\left(\mathrm{ZrO}_{2}\right)$ [8]. Il est ainsi amené à jouer un rôle important dans le domaine des électrolytes solides et des méthodes de production d'hydrogène par électrolyse de l'eau [9].

Malgré ces applications intéressantes, $\mathrm{Y}_{2} \mathrm{O}_{3}$ est relativement peu étudié. Ses propriétés mécaniques en particulier sont inconnues.

C'est dans le contexte de l'étude des propriétés plastiques de cet oxyde, que ce travail est effectué.

2. Echantillons d'oxyde d'yttrium. - La structure de $\mathrm{Y}_{2} \mathrm{O}_{3}$ peut être obtenue à partir de la structure fluorine $\left(\mathrm{CaF}_{2}\right)$ plus connue, dans laquelle on enlève le quart des anions de façon particulière [10]. En raison de ce déficit, la maille fluorine se dilate en se distordant légèrement pour donner la structure dite cubique type $C[11,12]$. Un grand nombre d'oxydes de terres rares possèdent cette structure à symétrie cubique centrée.

Le groupe d'espace est $\mathrm{Ia}_{3}\left(\mathrm{Th}_{7}\right)$. Il possède 16 molé- cules d' $\mathrm{Y}_{2} \mathrm{O}_{3}$ par maille, soit 80 atomes. Le paramètre cristallin est $a_{0}=10,604 \AA$ à $27^{\circ} \mathrm{C}$.

Les monocristaux d'oxyde d'yttrium sont difficiles à élaborer et seuls de petits monocristaux parallélépipédiques $\left(5 \times 2 \times 2 \mathrm{~mm}^{3}\right)$ ont été jusqu'alors employés pour des essais mécaniques en compression [13]. Ces expériences ont montré que les conditions limites pour lesquelles une déformation plastique évidente apparaissait, étaient atteintes pour une contrainte de $600 \mathrm{MPa}$ à $1120^{\circ} \mathrm{C}\left(0,51 T_{\mathrm{f}}\right)$. Pour des contraintes plus fortes ou des températures plus basses, l'oxyde d'yttrium, sollicité en compression, est fragile.

Les essais de microdureté parfois considérés comme des essais sous pression hydrostatique [14], en évitant les fractures multiples créées par des compressions ou des tensions, sont d'un grand intérêt pour l'étude des oxydes $[15,16]$. La possibilité d'effectuer ce genre d'essai en fonction de la température doit permettre de mettre en évidence une plasticité de ces matériaux dans une gamme de températures et de contraintes beaucoup plus grande que celle cernée par les essais classiques de compression, de traction ou de flexion.

3. Résultats expérimentaux. - Les essais de microdureté sur des monocristaux d' $\mathrm{Y}_{2} \mathrm{O}_{3}$ ont été réalisés avec un microduromètre Nikon pouvant travailler de la température ambiante à $1200^{\circ} \mathrm{C}$. Nous présentons quelques résultats préliminaires de microdureté uniquement réalisés avec un indenteur Vickers en saphir.

La plupart des essais ont été effectués sur une face d'orientation proche de (110) entre la température ambiante et $900^{\circ} \mathrm{C}$. Ils sont résumés sur la figure 1 qui représente la dureté en fonction de la température. 


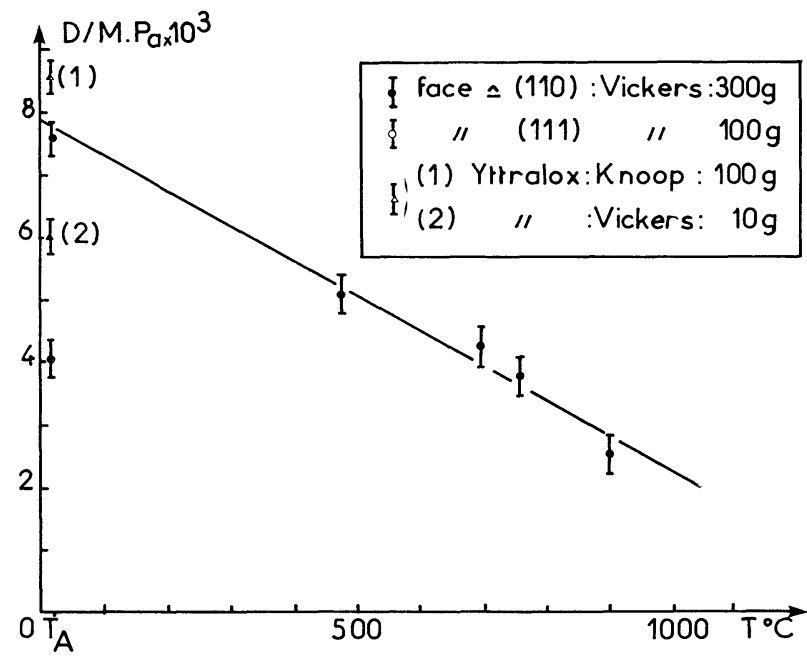

Fig. 1. - Microdureté Vickers, pour une charge de $300 \mathrm{~g}$, de $\mathrm{Y}_{2} \mathrm{O}_{3}$ en fonction de la température.

[Microhardness Vickers, with a load of $300 \mathrm{~g}$, of $\mathrm{Y}_{2} \mathrm{O}_{3}$, versus temperature.]

Cette courbe montre une décroissance quasi linéaire de la dureté en fonction de la température. Deux valeurs, obtenues sur de l'yttralox polycristallin à la température ambiante [7] par essais Vickers et Knoop, sont représentées à titre indicatif.

Les empreintes faites entre la température ambiante et $900^{\circ} \mathrm{C}$ ont été examinées en contraste interférentiel de Nomarski.

On a remarqué, autour de chaque empreinte, la présence de fractures et des marches de glissement visibles dès $480^{\circ} \mathrm{C}$ (Fig. 2). Elles deviennent très importantes pour $900{ }^{\circ} \mathrm{C}$ (Fig. 3). Pour des températures

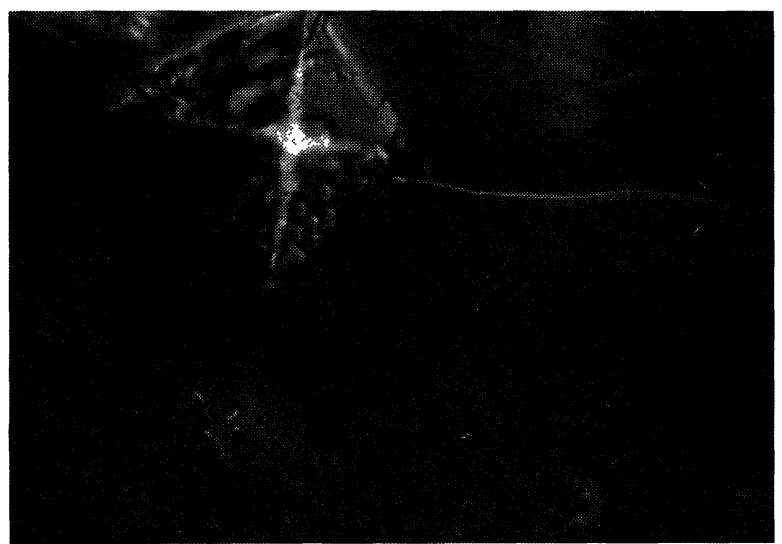

FIG. 2. - Indentation Vickers à $480^{\circ} \mathrm{C}$ sur une face (110) la flèche indique des marches de glissement.

[Vickers indentation at $480^{\circ} \mathrm{C}$ on a (110) face ; the arrow indicates glide steps.]

proches de l'ambiante le voisinage immédiat de l'empreinte est très confus du fait de l'importance des fractures et des éclats provoqués par l'indentation.

L'indexation de la face d'indentation et des directions

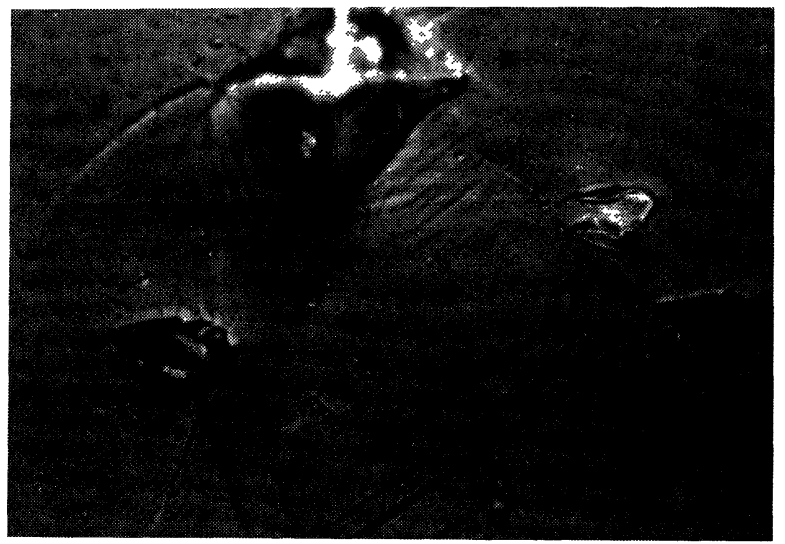

Fig. 3. - Indentation Vickers à $900^{\circ} \mathrm{C}$ sur ure face (110).

[Vickers indentation at $900^{\circ} \mathrm{C}$ on a (110) face.]

des marches de glissement par Laué est en accord avec les systèmes possibles de glissement suivant les plans (100) et (110) déjà détectés au cours d'essais de compression [13].

Sur la figure 1 est présenté un essai du même type

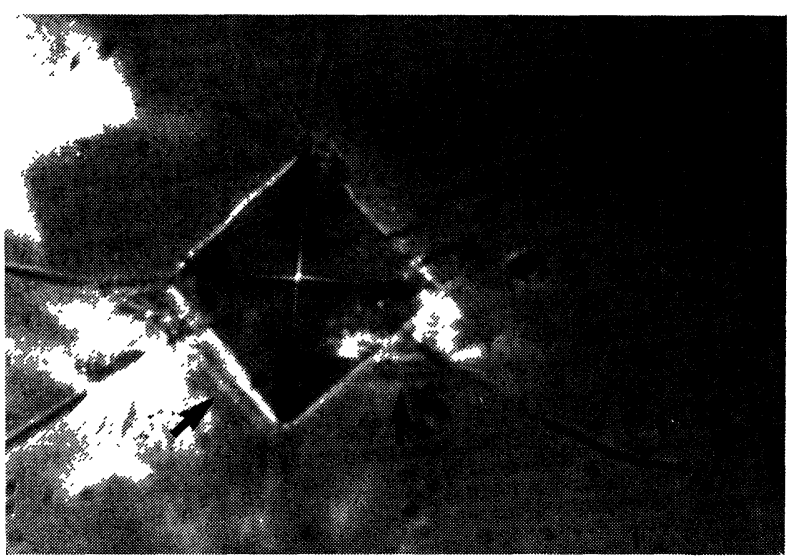

(a)

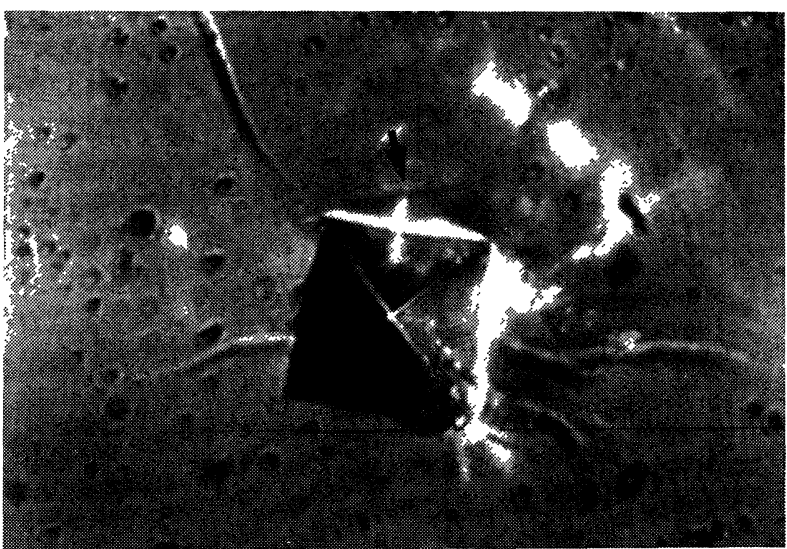

(b)

FIG. 4. - $-a$ ) et $b$ ) : Indentation Vickers à la température ambiante sur une face (111). Les flèches indiquent de possibles marches de glissement.

[a) and $b$ ) : Vickers indentation at room temperature on a (111) face. The arrows indicate possible glide plane emergence.] 
réalisé à température ambiante sur une face de clivage (111). La dureté apparaît plus faible. De nombreux essais ont été réalisés dans ces conditions. L'examen attentif des empreintes a parfois montré ce qui peut être considéré comme des marches de glissement (figure 4, $a$ et $b$ ). Ces observations sont cependant considérablement gênées par l'importance des phénomènes de fracture. Les traces observées sont dans des directions $<110>$ et $<112>$ et peuvent par conséquent, correspondre à des émergences de plans de glissement de type (001) ou (110).

Notons que les valeurs de la dureté, qui sont indiquées sur la figure 1 en fonction de la température, doivent être prises avec précaution. En effet, les fractures entourant les empreintes compliquent considérablement la répartition des contraintes et donc la réponse du matériau à l'indenteur.

Cependant, la plasticité de $\mathrm{Y}_{2} \mathrm{O}_{3}$ sur les figures 2 et 3 , ne fait aucun doute.

4. Discussion. - Des essais antérieurs de déformation plastique par compression ont montré que les plans (100) étaient les plans de glissement préférentiel de l'oxyde d'yttrium [13]. Les plans de type (110) plus difficilement actifs apparaissent comme les plans de glissement secondaire. Ces résultats sont surprenants dans la mesure où les plans de glissement primaire (100) ne contiennent pas le plus court vecteur de Burgers de la structure.

L'origine physique de ces plans peut être rattachée au fait qu'un cisaillement créant une faute d'empilement de très faible énergie y est possible [17]. Cette hypothèse est corroborée par une étude récente des dislocations présentes dans ces plans par microscopie électronique [18].

Les faces sur lesquelles ont été faites les empreintes ne permettent pas une détermination sans ambiguité du type de plan provoquant les marches d'émergence. Les traces peuvent provenir de plan (100) ou (110) ce qui est cohérent avec les résultats obtenus par d'autres modes de sollicitation.

Dans la mesure où le champ de contrainte provoqué par l'indenteur Vickers est assez mal connu, il est difficile de raisonner en terme de contrainte résolue dans les plans de glissement.

Sur la base de ces résultats, aspect des traces de glissement, et allure générale de la courbe de dureté, une déformation plastique faisant appel au même mécanisme sur la gamme de températures explorée est envisageable. Ces expériences et en particulier celles faites sur les faces (111) montrent que la plasticité de $\mathrm{Y}_{2} \mathrm{O}_{3}$ a lieu à des températures bien plus basses que ne le laissaient prévoir les essais de compression.

5. Conclusion. - Une étude de microdureté de $\mathrm{Y}_{2} \mathrm{O}_{3}$ monocristallin en fonction de la température est réalisée par indentation Vickers. De $480^{\circ} \mathrm{C}$ à $900^{\circ} \mathrm{C}$ la plasticité de cet oxyde se manifeste de façon évidente. Des empreintes réalisées sur une face (111) laissent prévoir une plasticité à la température ambiante.

\section{Bibliographie}

[1] Jones, T. P., J. Aust. Ceram. Soc. 101 (1974) 21.

[2] Kubachewski, O., Evans, E., Metal Thermo. Chem (Pergamon Press Oxford) 1956.

[3] Akerman, R. J. J. Chem. Phys. 403 (1964) 883.

[4] Jorgensen, J. P., Anderson, R. C., J. Am. Ceram. Soc. 50 11 (1967) 553.

[5] Borvkova, L. A., Lukin, E. S., Poluboyarinov, D. N., SAPOZHnikova, E. B., Ogneupory. 3511 (1970) 39.

[6] Brissette, L. A., Burnett, P. L., Spriggs, R. M., Vasilos, T., J. Am. Ceram. Soc. 49 (1966) 165.

[7] ANDerson, R. C., BArKer, J., Opt. Spectra 3 (1969) 57.

[8] ScotT, H. G., J. Mater Sci. 10 (1975) 1527.

[9] HagenMulleR, P., La Recherche 8 (1977) 756.

[10] ZAChARIASEN, W., Z. Kristallogr. 67 (1928) 455.
[11] Paton, M. G., Maslen, E. N., Acta Crystallogr. 193 (1965) 307.

[12] O'Connor, B. H., Valentine, T. M., Acta Crystallogr. (B) 25 (1969) 2140.

[13] Gaboriaud, R. J., Veyssière, P., Rabier, J., Boisson, M., J. Mater Sci. (1977) in press.

[14] The Science of hardness testing. (Wesbrook J. H. Conrad H.) Amer Soc. Met. (1973).

[15] Audouard, A,, Pelissiers, B., Castaing, J., J. Physique Lett. 38 (1977) L. 33.

[16] Lejus, A. M., Rev. inst. Htes Temp. et Refract. 13 (1976) 89.

[17] Boisson, M., Gaboriaud, R. J., J. Physique Lett. 38 (1977) L. 177.

[18] Gaboriaud, R. J., Denanot, M. F., Boisson, M., Grilhé, J., Phys. Status Solidi 1978 in press. 